\title{
Strong correlations in hot QCD
}

\section{T. Hatsuda*}

Department of Physics, The University of Tokyo, Tokyo 113-0033, Japan

E-mail: hatsuda@phys.s.u-tokyo.ac.jp

\begin{abstract}
After reviewing briefly the basic strategy of analyzing the RHIC data, recent lattice results of the non-pertubative/strong-coupling aspects of quark-gluon plasma probed primarily by the heavy quarks are presented. In particular, free energies of static quarks in various color channels, the Debye screening mass and the spatial string tension above the pseudo-critical temperature are discussed on the basis of the full QCD simulations with staggered quarks and with Wilson quarks. Current status of the equal-time Bethe-Salpeter wave functions and the spectral functions of the charmoniums in quenched QCD simulations is also summarized. It is argued that the hot plasma above the (pseudo-)critical temperature $T_{c}$ is still strongly interacting up to $T \sim(1.5-2) T_{c}$ so that hadronic bound states or resonances made of strange, charm and bottom quarks in certain channels may survive inside the plasma. An attempt to extract the spectral functions at finite $T$ in full QCD simulations is also mentioned.
\end{abstract}

XXIVth International Symposium on Lattice Field Theory

July 23-28, 2006

Tucson, Arizona, USA

\footnotetext{
* Speaker.
} 


\section{Introduction}

The ultimate goal of the heavy-ion experiments at RHIC and LHC are to create the quarkgluon plasma (QGP) and to study its properties in the laboratories. There have been already a plenty of data produced from RHIC [1]. When we analyse the data, we need to take into account the time evolution of the system. The relativistic hydrodynamics gives a suitable and successful framework for such purpose [2], as anticipated long time ago by Landau.

Here is what Landau wrote [3]:

(I) When two nucleons ${ }^{1}$ collide, a compound system is formed, and energy is released in a small volume $V$ subject to a Lorentz contraction in the transverse direction ${ }^{2}$. At the instant of collision, a large number of "particles" are formed; the "mean free path" in the resulting system is small compared with its dimensions, and statistical equilibrium is set up.

(II) The second stage of the collision consists in the expansion of the system. Here the hydrodynamic approach must be used, and the expansion may be regarded as the motion of an ideal fluid (zero viscosity and zero thermal conductivity). During the process of expansion the "mean free path" remains small in comparison with the dimensions of the system, and this justifies the use of the hydrodynamics. Since the velocities in the system are comparable with that of light, we must use not ordinary but relativistic hydrodynamics. Particles are formed and absorbed in the system throughout the first and second stages of the collision. The high density of energy in the system is of importance here. In this case, the number of particles is not an integral of the system, on account of the strong interaction between the individual particles.

(III) As the system expands, the interaction becomes weaker and the mean free path becomes longer. The number of particles appears as a physical characteristic when the interaction is sufficiently weak. When the mean free path becomes comparable with the linear dimensions of the system, the latter breaks up into individual particles. This may be called the "breakup" stage. It occurs with a temperature of the system of the order $T \approx \mu c^{2}$, where $\mu$ is the mass of the pion. (All temperatures are in energy units.)

Shown in Fig.1 is an outline of analyzing the data in relativistic heavy ion collisions together with a very similar case in the analyses of the cosmic microwave background radiation and its anisotropy. In hydrodynamical simulations of the relativistic heavy-ion collisions, we need several inputs: (i) the initial conditions at the time when local thermal equilibrium is established, (ii) the equation of state (the thermodynamic potential as a function of the temperature and the baryon chemical potential) which dictates the space-time evolusion of the relativistic fluid, and (iii) the freezeout (break-up) conditions at which the local thermal equilibrium is lost. Many attemps have been made to provide these inputs from various sources: e.g. the initial condition from the saturated gluon distribution (the color glass condensate), the equation of state from lattice QCD simulations and the freezout dynamics from parton recombinations.

\footnotetext{
${ }^{1}$ The nucleons here should be replaced by the nuclei in the present context.

${ }^{2}$ The word "transverse" here implies "longitudinal" in the modern notation.
} 


\begin{tabular}{|c|c|c|}
\hline & Big Bang & Little Bang \\
\hline Initial state & Inflation ? $\left(10^{-35} \mathrm{sec}\right)$ & Color glass ? $\left(10^{-24} \mathrm{sec}\right)$ \\
\hline Thermalization & Inflaton decay? & decoherence? \\
\hline Expansion & $\begin{aligned} R^{\mu \nu} & -\frac{1}{2} R g^{\mu \nu} \\
\quad= & 8 \pi G T^{\mu \nu}\end{aligned}$ & $\begin{array}{r}\partial_{\mu} T^{\mu \nu}=0 \\
\partial_{\mu} j_{\mathrm{B}}^{\mu}=0\end{array}$ \\
\hline Freezeout & $\begin{array}{l}(\mathrm{T}=1.95 \mathrm{~K} \text { neutrino }) \\
\mathrm{T}=2.73 \mathrm{~K} \text { photon }\end{array}$ & $\begin{array}{l}\mathrm{T}_{\text {chem }}=170 \mathrm{MeV} \\
\mathrm{T}_{\text {therm }}=120 \mathrm{MeV}\end{array}$ \\
\hline Observables & $\begin{array}{l}\text { CMB \& anisotropy } \\
\text { (C } \vee \text { B, CGB \& anisotropy) }\end{array}$ & $\begin{array}{l}\text { Flow \& its anisotropy, Hadrons, } \\
\text { Jets, leptons, photons }\end{array}$ \\
\hline $\begin{array}{c}\text { Key } \\
\text { Parameters }\end{array}$ & $\begin{array}{l}\text { 8 10 cosmological parameters } \\
\cdot \text { Initial density fluctuation } \\
\cdot \text { Cosmological const. } \Lambda \text { etc }\end{array}$ & $\begin{array}{l}\text { Plasma parameters } \\
\text { - Initial energy density } \\
\text { - thermalization time etc }\end{array}$ \\
\hline Evolution Code & CMBFAST & 3D-hydrodynamics \\
\hline
\end{tabular}

Figure 1: Comparison of the physics and analysis of the big bang (early universe) and the little bang (relativistic heavy-ion collisions). Taken from [1].

To study the signature of QGP in a quantitative manner, further details of QGP beyond its bulk properties are required. They include the transport coefficients, spectra of elementary excitations, static and dynamic responses to external probes or to internal impurities, etc. In recent years, lattice QCD techniques have been applied to those micro-properties of QGP. One of such examples is the calculation of the shear viscosity in quenched QCD by Nakamura and Sakai shown in Fig 2 [4]. In the phenomenological analysis of the RHIC data, it is still controversial whether QGP behaves as an ideal fluid or as a viscous fluid [5].

In the following, we will focus on a specific probe, the heavy quark(s) in QGP, from the point of view of the lattice QCD simulations. Such a probe is expected to unravel possible strong correlations of QGP and also is related to the measurement of dileptons ejected from the hot QCD matter created in relativistic heavy-ion collisions [6].

\section{Infinitely heavy quarks in QGP}

First, we summarize the recent attempts to probe QGP through infinitely heavy (= static) quarks on the lattice.

\subsection{Free enengies of two static quarks}

By appropriate gauge fixing (such as the Coulomb gauge fixing), one can define the free energies of two static quarks in various color channels through the correlation of the Polyakov lines 


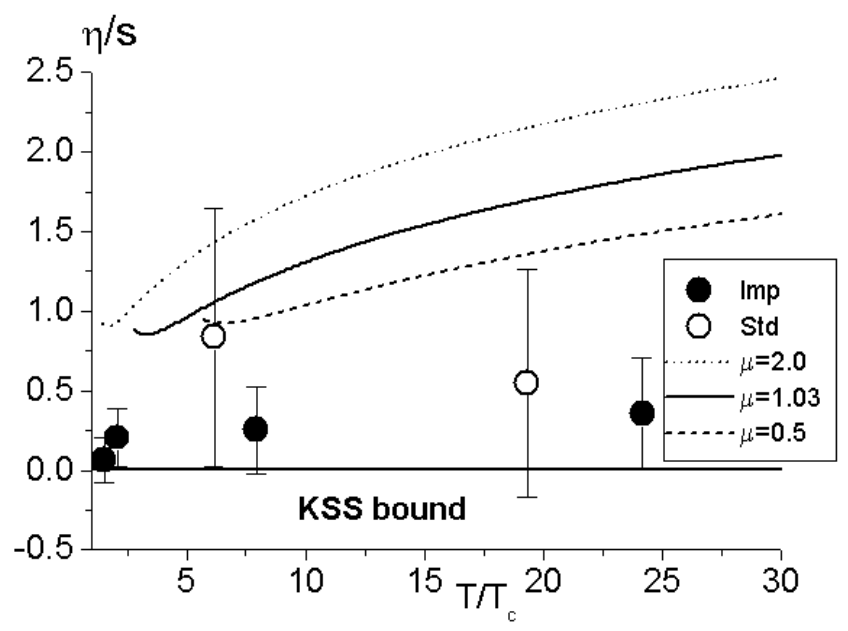

Figure 2: The shear viscosity $(\eta)$ divided by the entropy density $(s)$ at finite temperature in the quenched QCD on a $24^{3} \times 8$ lattice [4]. $\eta$ is obtained by fitting the spectral function associated with the imaginary-time correlation of the energy-momentum tensor. The solid and dashed lines are the results of the weak coupling calculation in thermal QCD for different choices of the renormalization point $\mu$ in the unit of $T$. The KSS bound $\left(=(4 \pi)^{-1}\right)$ shows the value in the strong coupling limit of the $N=4$ supersymmetric Yang-Mills theory.

$\Omega$ and $\Omega^{\dagger}[7]$. For the color singlet $Q \bar{Q}$ channel (1), the color octet $Q \bar{Q}$ channel (8), the color anti-triplet $Q Q$ channel $\left(\mathbf{3}^{*}\right)$, and the color sextet $Q Q$ channel (6), we have

$$
\begin{aligned}
e^{-F_{\mathbf{1}}(r, T) / T} & =\frac{1}{3}\left\langle\operatorname{Tr} \Omega^{\dagger}(\mathbf{x}) \Omega(\mathbf{y})\right\rangle, \\
e^{-F_{\mathbf{8}}(r, T) / T} & =\frac{1}{8}\left[\left\langle\operatorname{Tr} \Omega^{\dagger}(\mathbf{x}) \operatorname{Tr} \Omega(\mathbf{y})\right\rangle-\frac{1}{3}\left\langle\operatorname{Tr} \Omega^{\dagger}(\mathbf{x}) \Omega(\mathbf{y})\right\rangle\right], \\
e^{-F_{\mathbf{6}}(r, T) / T} & =\frac{1}{12}[\langle\operatorname{Tr} \Omega(\mathbf{x}) \operatorname{Tr} \Omega(\mathbf{y})\rangle+\langle\operatorname{Tr} \Omega(\mathbf{x}) \Omega(\mathbf{y})\rangle], \\
e^{-F_{3^{*}}(r, T) / T} & =\frac{1}{6}[\langle\operatorname{Tr} \Omega(\mathbf{x}) \operatorname{Tr} \Omega(\mathbf{y})\rangle-\langle\operatorname{Tr} \Omega(\mathbf{x}) \Omega(\mathbf{y})\rangle],
\end{aligned}
$$

where $r=|\mathbf{x}-\mathbf{y}|$.

Shown in Fig 3 is the free energy between a static quark $Q$ and a static anti-quark $\bar{Q}$ in the color singlet channel below and above the pseudo-critical temperature $T_{c}$ in the (2+1)-flavor QCD. The p4-improved staggered quark action on a $16^{3} \times 4$ lattice is adopted [8]. The light quark mass is chosen to be $m_{q}=0.1 m_{s}$ with $m_{s}$ being the strange quark mass. The overall constant of the free energies at each $T$ is adjusted to the zero temperature potential (shown by the solid line) at their shortest distances. The free energy is screened at long distances below $T_{c}$ by the string breaking due to dynamical quark-anti-quark pairs at finite $T$. The screening above $T_{c}$ may be interpreted as the standard Debye screening.

Shown in the left panel of Fig 4 are the free energies of a static quark-anti-quark pair in the color singlet $\left(V_{1}(r, T)\right)$ and the color octet $\left(V_{8}(r, T)\right)$ channels. The right panel of Fig.4 shows the 


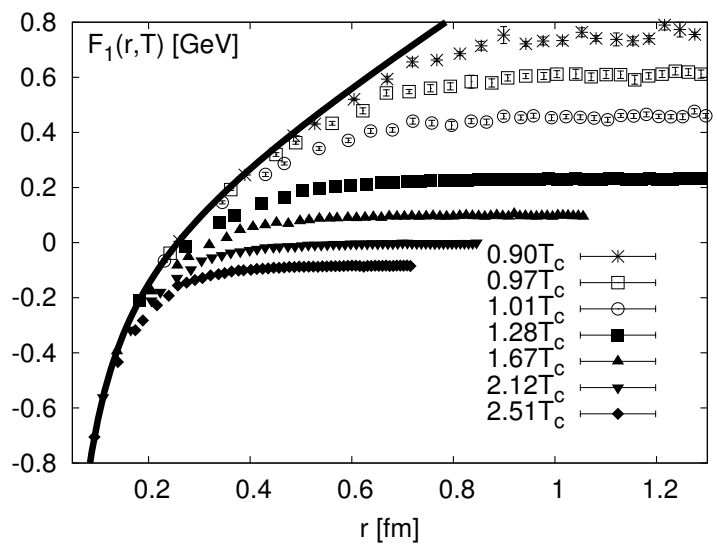

Figure 3: Free energy of a heavy quark and an anti-quark in the color-singlet channel separated by distance $r$ [8]. Simulations are done in the (2+1)-flavor QCD with the p4-improved staggered quark action on a $16^{3} \times 4$ lattice. The Coulomb gauge fixing is adopted. The light quark mass is chosen to be $m_{q}=0.1 m_{s}$.

free energies of a static quark-quark pair in the color anti-triplet $\left(V_{3^{*}}(r, T)\right)$ and the color sextet $\left(V_{6}(r, T)\right)$ channels. The results are obtained from 2-flavor QCD with the clover-improved Wilson quark action [9]. The quark mass is chosen so that $m_{\pi} / m_{\rho}=0.65$ in this figure. The overall constant of the free energy is adjusted to be zero at large distance. The sign and the magnitude of the free energies in different channels can be well fitted by the screened Coulomb form

$$
V_{M}(r, T)=C_{M} \frac{\alpha_{\mathrm{eff}}(T)}{r} e^{-m_{D}(T) r},
$$

with the Casimiar factor $C_{M}(=-4 / 3,1 / 6,-2 / 3,1 / 3)$ for $M=\mathbf{1}, \mathbf{8}, \mathbf{3}^{*}, \mathbf{6}$, respectively. $\alpha_{\mathrm{eff}}(T)$ and $m_{D}(T)$ are the effective coupling and the Debye screening mass, respectively. These parameters are assumed to be channel independent.
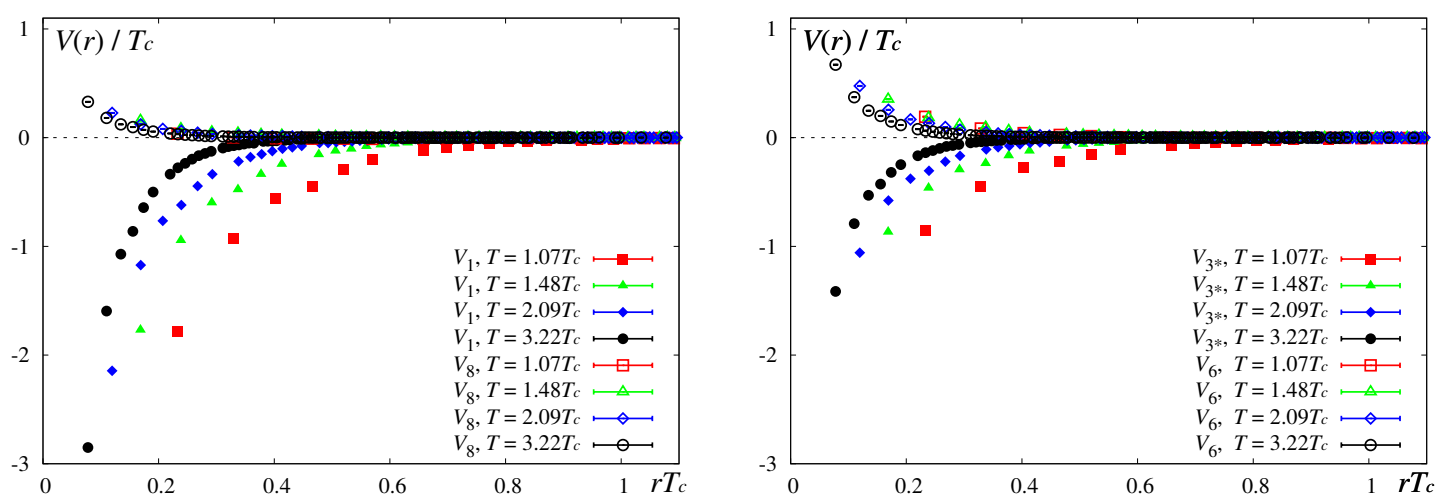

Figure 4: (Left panel) The ree energies of a static quark-anti-quark pair in the color singlet $\left(V_{1}(r)\right)$ and the color octet $\left(V_{8}(r)\right)$ channels. (Right panel) The free energies of a static quark-quark pair in the color anti-triplet $\left(V_{3^{*}}(r)\right)$ and the color sextet $\left(V_{6}(r)\right)$ channels. The simulations are done for 2-flavor QCD with the clover improved Wilson quark action [9]. The quark mass is chosen so that $m_{\pi} / m_{\rho}=0.65$. 


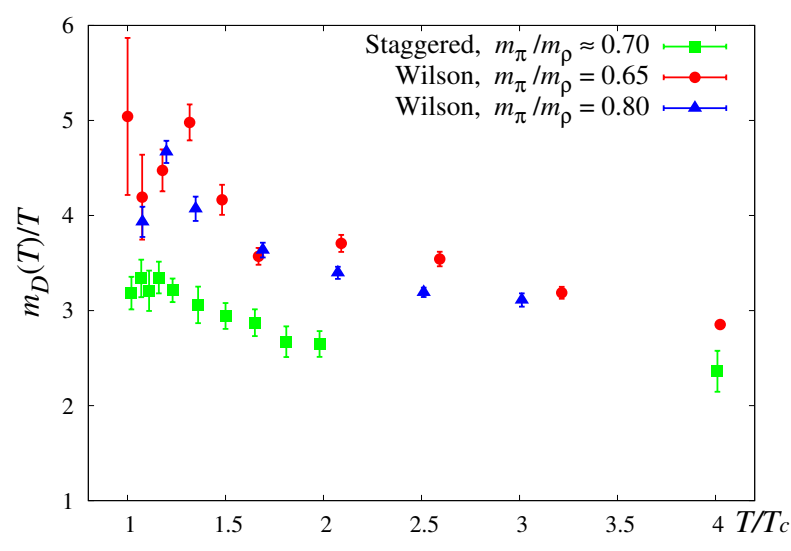

Figure 5: A comparison between the Debye mass calculated in 2-flavor QCD on a $16^{3} \times 4$ lattice with the clover-improved Wilson quark action (blue and red points) [9] and that with the p4-improved staggered quark action (green points) [11].

\subsection{Debye screening mass}

In the leading order (LO) of the thermal pertubation theory, the Debye screening mass between static quarks is simply given by $m_{D}^{\mathrm{LO}}(T)=\left(1+N_{f} / 6\right)^{1 / 2} g(T) T$ in the presence of the massless dynamical quarks with $N_{f}$-flavors. However, in the next-to-leading order, the magnetic sector of QCD enters into the game, and the Debye mass becomes non-perturbative [10]. Therefore, it is important to carry out full QCD simulations of $m_{D}(T)$ which is one of the key quantities to characterize the plasma.

Shown in Fig.5 is a comparison between the Debye mass calculated in 2-flavor QCD on a $16^{3} \times 4$ lattice with the clover-improved Wilson quark action (blue and red points) [9] and that with the p4-improved staggered quark action (green points) [11]. The overall magnitude of $m_{D}(T)$ and its $T$-dependence are qualitatively consistent with those expected from the hard thermal loop resummation supplmented by a "reasonable" value of the magnetic screening mass. Systematic discrepancy of about $20 \%$ between the two fermion formulations should be resolved in the future by making simulations with smaller lattice spacings (larger temporal sites). After eliminating such systematic errors, one would be able to make more quantitative statement.

\subsection{Spatial string tension}

Another observable which requires non-perturbative calculation is the spatial string tension $\sigma_{s}$ obtained from the spatial Wilson loop at high temperature [12]. According to the idea of the dimensional reduction at high temperature together with the confining feature of the 3-dimensional Yang-Mills theory, one may expect that the spatial Wilson loop has an area law in both quenched and full QCD at high temperature.

The left panel of Fig.6 shows $T / \sqrt{\sigma_{s}}$ as a function of $T / T_{c}$ in quenched QCD $\left(N_{f}=0\right)$ and in (2+1)-flavor QCD with the p4-improved staggered quark action on the $16^{3} \times 4$ lattice [13]. The quark mass in the $N_{f}=2+1$ case is chosen to be $m_{q} / m_{s}=0.1$. The dashed line is a fit using the form $\sqrt{\sigma_{s}}(T)=c g^{2}(T) T$. The right panel of Fig 6 shows $\sqrt{\sigma_{s}} / T$ as a function of $T / T_{c}$ in 2-flavor 

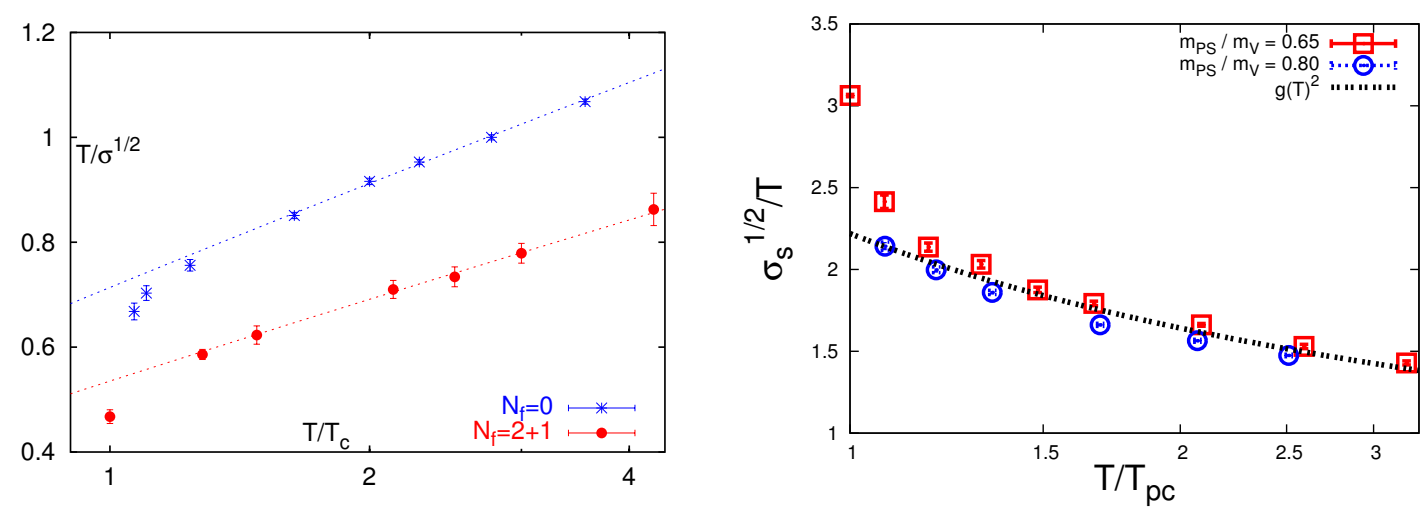

Figure 6: (Left panel) Temperature dependence of $T / \sqrt{\sigma_{s}}$ in $N_{f}=0$ and $N_{f}=2+1$ QCD with the p4improved staggered quark action on a $16^{3} \times 4$ lattice [13]. The quark mass in $N_{f}=2+1$ case is $m_{q} / m_{s}=$ 0.1. (Right panel) Temperature dependence of $\sqrt{\sigma_{s}} / T$ in 2-flavor QCD with clover-improved Wilson quark action on a $16^{3} \times 4$ lattice [14]. The quark masses correspond to $m_{\pi} / m_{\rho}=0.65$ and 0.8 .

QCD with clover-improved Wilson quark action on the $16^{3} \times 4$ lattice [14]. The quark masses correspond to $m_{\pi} / m_{\rho}=0.65$ and 0.8 . The dashed line is again a fit using the form above. In both cases, the approximate linear increase of $\sqrt{\sigma_{s}}$ is clearly seen up to a relatively weak (possibly logarithmic) correction. As for the comparison between the lattice data and the result of dimensionally reduced effective theory, see Fig.5 of [14].

\section{Heavy quarks with finite mass in QGP}

Although the infinitely heavy quarks give us useful information on the plasma properties as seen in the previous section, the heavy quarks in the real world (such as the charm and bottom) have finite masses and may receive substantial kick from the plasma-constituents which have typical energy of about $3 T$. Therefore, it is important to examine the dynamical correlations (both spatial and temporal) of the heavy quarks inside QGP.

\subsection{Charmonium wave function}

An pioneering attempt to study the $J / \Psi$ wave function at finite temperature in quenched QCD on an anisotropic lattice was performed by Umeda et al. [15]. They have studied the equal-time Bethe-Salpeter wave function at finite $T$ in the Coulomb gauge:

$$
w(\vec{r}, \tau)=\sum_{\vec{x}}\left\langle\bar{q}(\vec{x}, \tau) \gamma_{i} q(\vec{x}+\vec{r}, \tau) \mathscr{O}^{\dagger}(0)\right\rangle,
$$

where $\mathscr{O}$ is a source to creat $J / \Psi$ at $\tau=0$. In Fig.7, the normalized wave function, $\phi(\vec{r}, \tau)=$ $w_{i}(\vec{r}, \tau) / w_{i}(\overrightarrow{0}, \tau)$ is plotted below and above $T_{c}$ for various time slices. The black dashed line is the wave function prepared at $\tau=0$. For free quarks without gauge interactions, the wave function becomes broad as the imaginary time $\tau$ increases. On the other hand, the wave functions of the interacting system has little $\tau$ dependence. More strikingly, the wave function at $T=1.52 T_{c}$ is almost identical to that below $T_{c}$, which suggests that $J / \Psi$ may survive as a bound state even if the system is in the deconfined phase. 


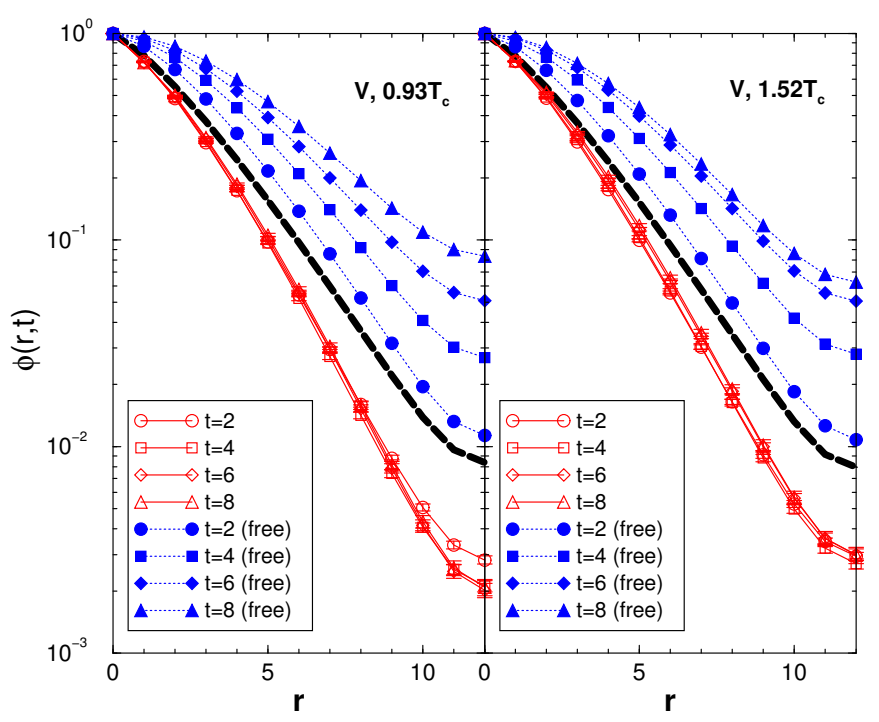

Figure 7: The equal-time Bethe-Salpeter wave function normalized at the origin as a function of the spatial separation $r$ between the charm and the anti-charm. Blue (red) points correspond to free (interacting) quarks. The black dashed line is the wave function initially prepared at $\tau=0$. Note that $t \equiv \tau$ in this figure.

\subsection{Charmonium spectral function}

The spectral functions of hadronic correlators give us another information on mesonic and baryonic modes at finite $T$. Such studies date back to the seminal works [16, 17] in which the existence of the hadronic modes in QGP was suggested for the first time.

The spectral function $\sigma(\omega, \vec{p})$ for hadronic correlation may be defined through the spectral decomposition:

$$
D(\tau, \vec{p})=\int_{-\infty}^{+\infty} \frac{\mathrm{e}^{-\tau \omega}}{1 \mp \mathrm{e}^{-\omega / T}} \sigma(\omega, \vec{p}) d \omega \quad\left(0 \leq \tau<T^{-1}\right) .
$$

Reconstructing a continuous function $\sigma(\omega, \vec{p})$ from discrete and finite number of lattice data $D(\tau, \vec{p})$ is a typical ill-posed problem. The maximum entropy method (MEM) provides an efficient and powerful approach to avoid the problem as reviewed in [18, 19]: one can obtain a unique $\sigma$ from the lattice data $D$ without making a priori parameterization. Also, statistical significance of the resultant $\sigma$ can be evaluated. In MEM, the most probable $\sigma$ given lattice data $D$ is obtained by maximizing the conditional probability $P[\sigma \mid D] \propto e^{\alpha S-L}$, where $L$ is the standard likelihood function and $S$ is the Shannon-Jaynes information entropy:

$$
S=\int_{0}^{\infty}\left[\sigma(\omega)-m(\omega)-\sigma(\omega) \log \left(\frac{\sigma(\omega)}{m(\omega)}\right)\right] d \omega
$$

The statistical significance (error) of the resultant $\sigma$ is estimated by the second variation, $\left(\frac{\delta}{\delta \sigma}\right)^{2} P[\sigma \mid D]$. The default model $m(\omega)$ in Eq.(3.3) may be chosen so that the MEM errors become minimum. The final result is given by a weighted average over the parameter $\alpha$ as $\sigma(\omega, \vec{p})=\int \sigma_{\alpha}(\omega, \vec{p}) P[\alpha \mid D m] d \alpha$, where $\sigma_{\alpha}(\omega, \vec{p})$ is obtained by minimizing $P[\sigma \mid D]$ for a fixed $\alpha$. 

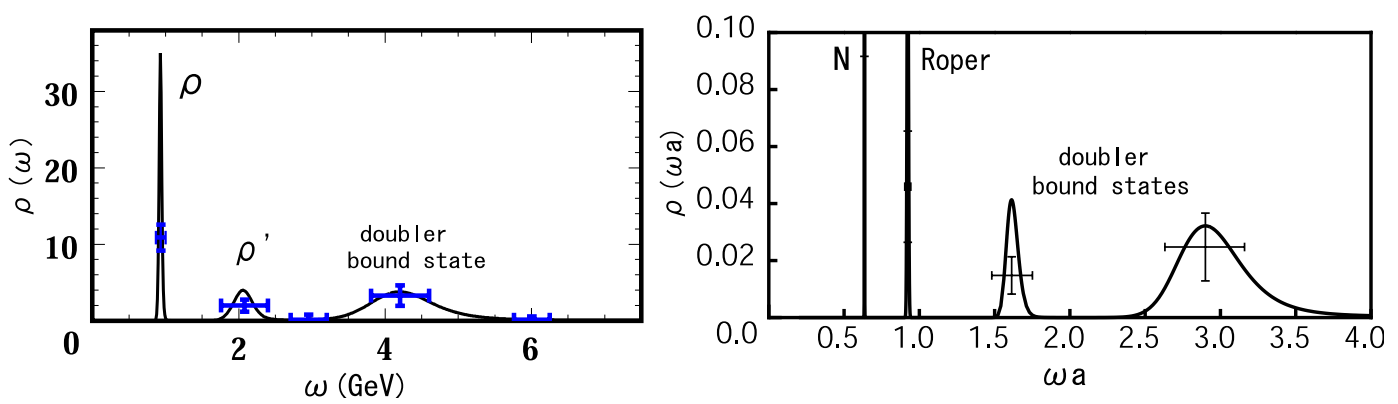

Figure 8: (Left panel) The dimensionless spectral function $\rho(\omega)=\sigma(\omega, \overrightarrow{0}) /\left(3 \omega^{2}\right)$ of the vector meson extracted from quenched QCD simulations on a $20^{3} \times 24$ lattice with the lattice spacing $a=0.0847 \mathrm{fm}$ and the hopping parameter $\kappa=0.1557$.[20]. (Right panel) The dimensionless spectral function $\rho(\omega)=$ $\sigma(\omega, \overrightarrow{0}) / \omega^{5}$ in the nucleon channel extracted from quenched QCD simulations on a $32^{4}$ lattice with $\beta=6.0$ $(a \simeq 0.093 \mathrm{fm})$ [21]. The hopping parameter is take to be $\kappa=0.1550$. The horizontal axis denotes a dimensionless frequency $\omega a$.

To see how it works, we show, in the left panel of Fig. 8 , the spectral image of the vector meson at rest $(\vec{p}=\overrightarrow{0})$ extracted from the quenched QCD data [20]. The first (second) peak corresponds to the ground (excited) vector meson. On the other hand, the highest peak corresponds to a bound state of Wilson doublers. Shown in the right panel of Fig. 8 is the spectral function in the nucleon channel extracted from the quenched QCD data [21]. The first (second) peak corresponds to the nucleon and the Roper resonance, while the higher two peaks correspond to the bound states of Wilson doublers.

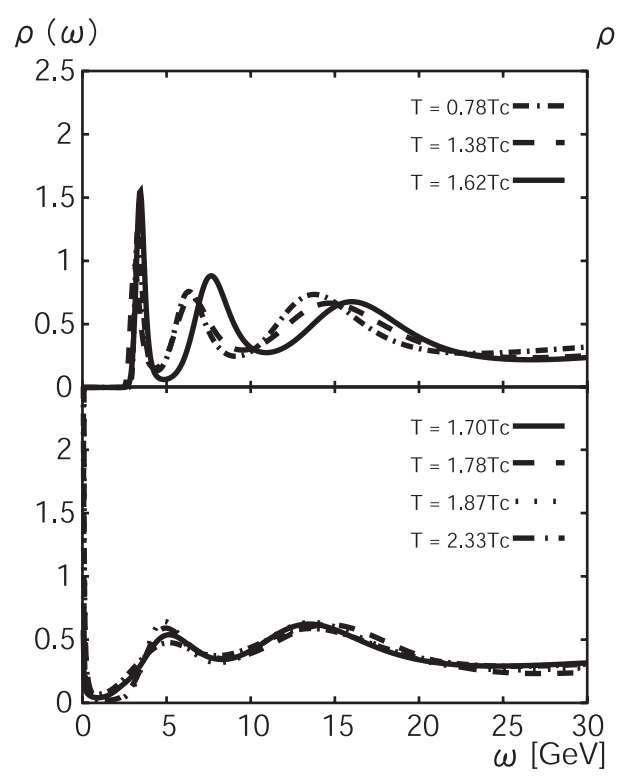

(a)

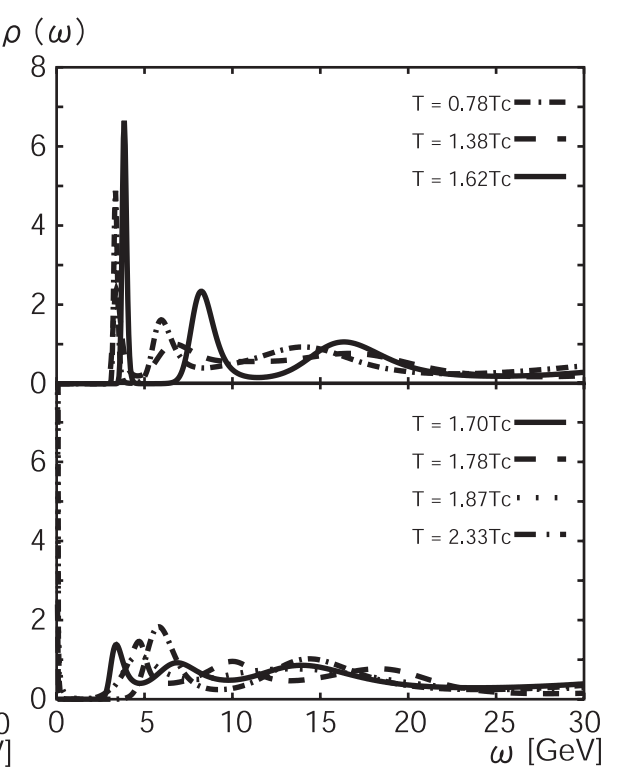

(b)

Figure 9: Spectral functions measured in quenched QCD simulations on an anisotropic lattice with the spatial volume $(1.25 \mathrm{fm})^{3}$ for (a) the $J / \Psi$ channel $\left(\rho(\omega)=\sigma(\omega, \overrightarrow{0}) /\left(3 \omega^{2}\right)\right)$ and for (b) the $\eta_{c}$ channel $\left(\rho(\omega)=\sigma(\omega, \overrightarrow{0}) / \omega^{2}\right)[22]$. 


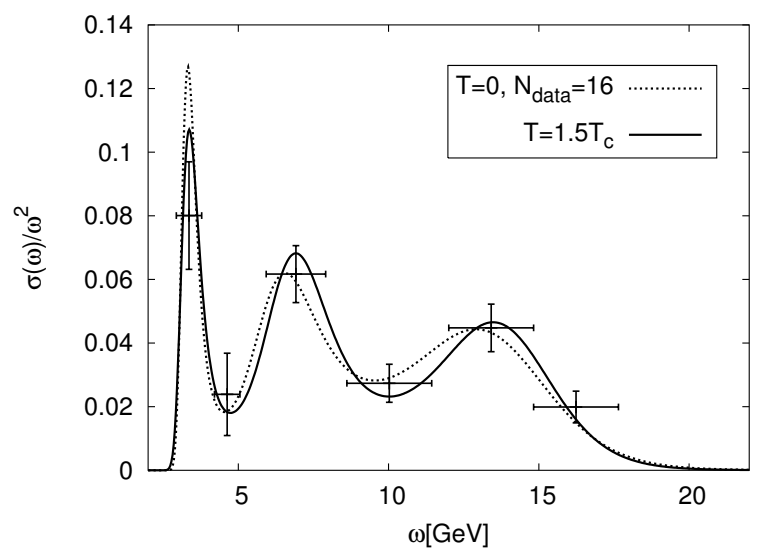

Figure 10: Spectral functions measured in quenched QCD simulations on an anisotropic lattice with the spatial volume $(1.34 \mathrm{fm})^{3}$ for the $\eta_{c}$ channel $\left(\sigma(\omega, \overrightarrow{0}) / \omega^{2}\right)$ [25].

The above MEM approach has been soon applied to charmoniums at finite $T[22,23,24]$ and it was found that the low-lying s-wave resonances such as $J / \Psi$ and $\eta_{c}$ survive above $T_{c}$ as distinct peaks with not much modification of their pole positions. For example, In ref. [22], quenched QCD simulations were performed with $\beta=7.0$ on $32^{3} \times(96-32)$ anisotropic lattices with a renormalized anisotropy $\xi=a_{s} / a_{t}=4.0$. (They correspond to $a_{t}=a_{s} / 4=9.75 \times 10^{-3} \mathrm{fm}$ and $L_{s}=1.25 \mathrm{fm}$.) Shown in Fig. 9 is the dimensionless spectral functions for $J / \Psi$ and $\eta_{c}$ below and above $T_{c}$. The figure indicates that both resonaces survive up to around $1.62 T_{c}$ and disappear at higher temperatures.

Recently, the quenched simulations with similar lattice size as [22] were reported by Jakovac et al. [25], where $24^{3} \times(160-34)$ anisotropic lattices are adopted with $\xi=4.0, a_{t}=a_{s} / 4=0.056 \mathrm{fm}$ and $L_{s}=1.34 \mathrm{fm}$. Shown in Fig.10 is the spectral function for $\eta_{c}$ (pseudo-scalar charmonium). The result indicates that $\eta_{c}$ survives at least up to $1.5 T_{c}$, which is consistent with the previous results.

\subsection{Further questions}

Except for trying to increase the statistics to sharpen the resolutions of the spectral function in MEM, there are several issues to be addressed about the results shown in Figs.9]10. For example, (i) is the spatial size of the lattice large enough? [26], and (ii) what about the case for full QCD? [28] Also, we would like to know what will happen for the charmoniums moving inside the medium [29, 30], for the lighter quark masses (such as u, d, s quarks) [31], and for the heavier quark mass (such as b) [32].

Let us briefly comment on some of the points mentioned above.

\subsubsection{Spatial lattice size}

One may wonder if charmonium above $T_{c}$ found in quenched simulations is an artifact due to the small spatial volume, $L_{s}=1.25-1.34 \mathrm{fm}$. To clarify this point, Iida et al. [26] has carried out quenched simulations with a periodic boundary condition (PBC) and an anti-periodic boundary condition (APBC) for the quarks on $16^{3} \times(26-14)$ anisotropic lattices with $\xi=a_{s} / a_{t}=4.0$ and 

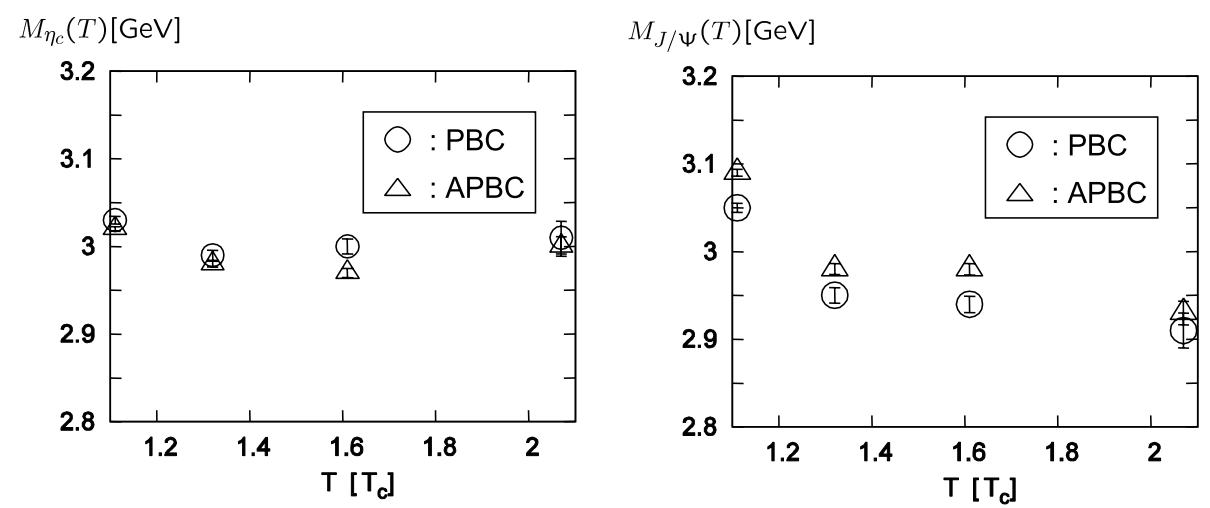

Figure 11: The pole masses of $J / \Psi$ and $\eta_{c}$ in the quenched QCD with the periodic (circle) and anti-periodic (triangle) boundary conditions [26]. Anisotropic lattices $(\xi=4)$ with the size $16^{3} \times(26-14)$ and $L_{s}=1.55$ fm are used.

$L_{s}=1.55 \mathrm{fm}$. The pole masses are obtained from the standard effective mass plot (not from MEM). Fig.11 shows that the pole masses do not have significant dependence on the boundary conditions above $T_{c}$. This implies that the bound states in the hot QCD plasma are spatially compact objects, which is consistent with the result of the spatial wave function in Fig.7.

\subsubsection{Full QCD}

In quenched QCD, only gluons are thermally excited in the plasma, while, in full QCD, both quarks and gluons are thermally active. Therefore, one might consider that the bound states above $T_{c}$ found in the quenched QCD may be easily destroyed by the thermal quarks in full QCD. However the situation is not that simple. In fact, when dynamical quarks are included, the critical temperature of the phase transition is substantially reduced from the quenched case. Therefore, the total number of thermal plasma-constituents becomes even smaller in full QCD and hence the net thermal dissociation rate of the hadronic resonances above $T_{c}$ as a function of $T / T_{c}$ could be smaller in full QCD [27]. Anyhow, it is truly necessary to carry out full QCD simulations to draw definite conclusion on this point.

Aarts et al. has recently reported an exploratory study with 2-flavor QCD on $8^{3} \times(32-16)$ anisotropic lattices with $\xi=6$ [28]. Their spatial lattice volume is $L_{s} \sim 1.2 \mathrm{fm}$ and the light quark mass corresponds to $m_{\pi} / m_{\rho} \sim 0.5$. Shown in Fig.12 are the spectral functions for the $\eta_{c}$ (pseudoscalar) and the $J / \Psi$ (vector) channels. The results suggest that they are at least bound at $T \sim 1.3 T_{c}$ and are eventually destroyed at $T \sim 2 T_{c}$ in full QCD.

\subsubsection{Heavier quark}

Let us go back the case of quenched QCD. A pilot study on the spectral functions of the bottomoniums in quenched QCD at finite $T$ was reported in [32] by using the same lattice set up as [23]. As shown in Fig.13, the spectral structure of pseudo-scalar meson $\eta_{b}$ does not receive modifications at $T=1.5 T_{c}$ while that of the scalar meson $\chi_{b 0}$ receives significant change at the same temperature. 

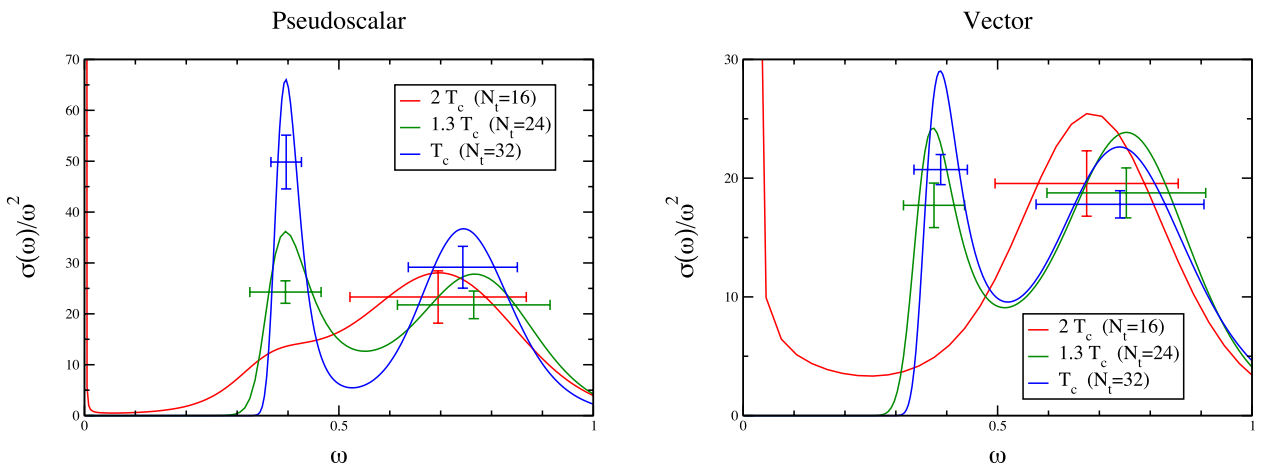

Figure 12: Spectral functions in the pseudo-scalar and vector channels in 2-flavor QCD on $8^{3} \times(32-16)$ anisotropic lattices with the renormalized anisotropy $\xi=6$ [28]. The spatial lattice volume is $L_{s} \sim 1.2 \mathrm{fm}$ and the light quark mass corresponds to $m_{\pi} / m_{\rho} \sim 0.5$.

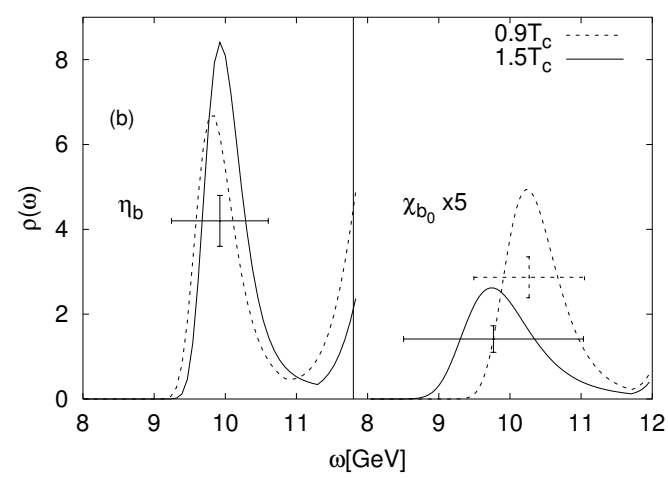

Figure 13: The dimensionless spectral function at finite $T$ for bottomoniums (pseudo-scalar $\eta_{b}$ and scalar $\chi_{b 0}$ ) extracted from the quenched QCD simulations [32].

\subsubsection{Lighter quark}

One may wonder if there also exist sharp resonances made of lighter quarks ( $\mathrm{u}, \mathrm{d}$ and $\mathrm{s}$ ) above $T_{c}$. An early attempt along this line is shown in Fig 14 where the spectral functions of the $s \bar{s}$ mesons in the scalar (S), pseudo-scalar (PS), vector (V) and axial-vector (AV) channels are presented. This is based on the quenched QCD simulations with the Wilson quark at $T / T_{c}=1.38$ [31]. The quark mass is chosen to reproduce the experimental $\phi$ meson mass at $T=0$. Other lattice set up is the same as [22]. One finds that spectral functions in all channels have degenerate peaks around 2.5 $\mathrm{GeV}$ at $T / T_{c}=1.38$ (2.4 times the $\phi$-meson mass at $T=0$ ). Also, these peaks disappear at higher temperatures e.g. at $T / T_{c}=1.87$ [31]. (Note that second and third peaks in the figure are likely to be the doubler bound states.) This results indicate that relatively heavy but sharp resonances composed of light quarks may also exit above $T_{c}$. How the masses of these resonances behave near $T_{c}$ is an interesting open problem to be explored in quenched and full QCD. 


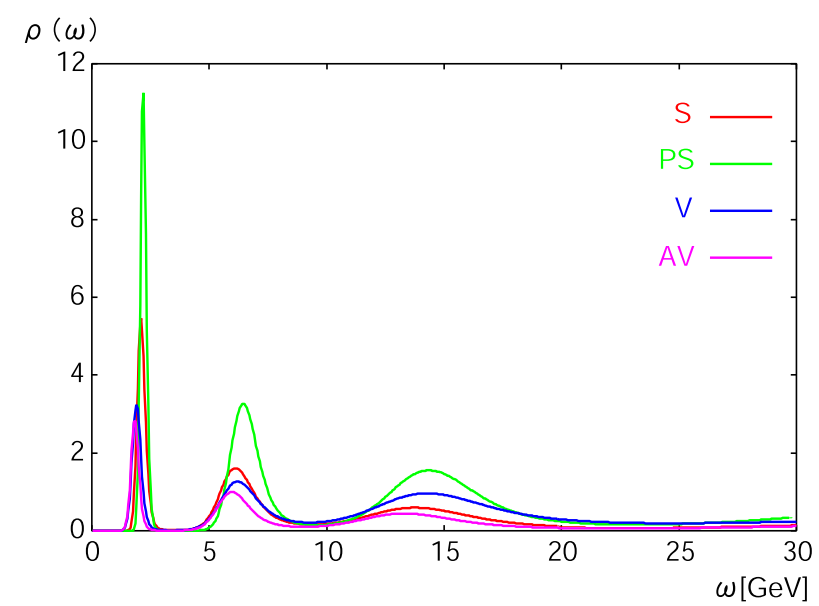

Figure 14: The dimensionless spectral function measured in quenched QCD simulations on an anisotropic lattice for the $s \bar{s}$ mesons at $T / T_{c}=1.38$ [31].

\section{4. summary}

In this article, we have made a brief review on the heavy quarks in the quark-gluon plasma. The lattice QCD simulations give us useful information on the free energies of static quarks with fixed separation and with various color combinations. The Debye mass and the spatial string tension are the fundamental quantities which characterize the plasma. They cannot be calculable in the thermal perturbation theory without non-perturbative inputs from the magnetic sector of QCD. The direct numerical simulations of these quantities in full QCD simulations are therefore very useful. A comparison of the results between different fermion actions (the p4-improved staggered quark and the clover-improved Wilson quark action) was given.

We have also summarized the current knowledge of the charmoniums inside the gluon plasma on the basis of the data taken in quenched QCD simulations. The results of the spatial wave function and the spectral functions of $J / \Psi$ and $\eta_{c}$ give a coherent picture that they survive as compact objects in the gluon plasma up to $T \sim(1.5-2) T_{c}$. An attempt toward full QCD simulations of the charmonium in the quark-gluon plasma has been started. To gain better understanding of the origin of the hadronic modes in the quark-gluon plasma, systematic numerical studies with heavier and lighter quark masses are crucial. Also theoretical studies to bridge the lattice data and the phenomenological models (e.g. [33, 34, 35, 36]) are important. Eventually, the lattice predictions should be compared with the RHIC and LHC data by the aid of the relativistic hydrodynamics.

Acknowledgements: This work was partially supported by Japanese MEXT Grant No. 18540253. The author thanks T. Umeda and K. Petrov for useful information. 


\section{References}

[1] K. Yagi, T. Hatsuda and Y. Miake, Quark-Gluon Plasma, (Cambridge Univ. Press, Cambridge 2005).

[2] T. Hirano, Eur. Phys. J. A 29 (2006) 19 [nucl-th/0511036].

[3] S. Z. Belensky and L. D. Landau, Ups. Fiz. Nauk. 56 (1955) 309, reprinted in Collected Papers of L.D. Landau ed. D.T. ter Haar, (Gordon \& Breach, New York,1965) 665.

[4] S. Sakai and A. Nakamura, PoS LAT2005 (2006) 186 [hep-lat/0510100].

[5] T. Hirano, U. W. Heinz, D. Kharzeev, R. Lacey and Y. Nara, Phys. Lett. B 636 (2006) 299 [nucl-th/0511046].

[6] T. Matsui and H. Satz, Phys. Lett. B 178 (1986) 416.

T. Hashimoto, K. Hirose, T. Kanki and O. Miyamura, Phys. Rev. Lett. 57 (1986) 2123.

[7] S. Nadkarni, Phys. Rev. D 33 (1986) 3738; 34 (1986) 3904.

[8] K. Petrov, hep-lat/0610041.

[9] Y. Maezawa, S. Ejiri, T. Hatsuda, N. Ishii, N. Ukita, S. Aoki and K. Kanaya, hep-lat/0610013.

[10] U. Kraemmer and A. Rebhan, Rept. Prog. Phys. 67 (2004) 351 [hep-ph/0310337].

[11] O. Kaczmarek and F. Zantow, Phys. Rev. D 71 (2005) 114510 [hep-lat/0503017].

[12] Y. Schroder and M. Laine, PoS LAT2005 (2006) 180 [hep-lat/0509104].

[13] T. Umeda, hep-lat/0610019.

[14] N. Ukita, S. Ejiri, T. Hatsuda, N. Ishii, Y. Maezawa, S. Aoki and K. Kanaya, hep-lat/0610038.

[15] T. Umeda, R. Katayama, O. Miyamura and H. Matsufuru, Int. J. Mod. Phys. A 16 (2001) 2215 [hep-lat/0011085]. T. Umeda, hep-lat/0610030.

[16] T. Hatsuda and T. Kunihiro, Phys. Rev. Lett. 55 (1985) 158.

[17] C. DeTar, Phys. Rev. D 32 (1985) 276.

[18] M. Jarrell and J.E. Gubernatis, Phys. Rep. 269 (1996) 133.

[19] M. Asakawa, T. Hatsuda and Y. Nakahara, Prog. Part. Nucl. Phys. 46 (2001) 459.

[20] Y. Nakahara, M. Asakawa and T. Hatsuda, Phys. Rev. D 60 (1999) 091503.

[21] K. Sasaki, S. Sasaki and T. Hatsuda, Phys. Lett. B623 (2005) 208.

[22] M. Asakawa and T. Hatsuda, Phys. Rev. Lett. 92 (2004) 012001; J. Phys. G30 (2004) S1337.

[23] S.Datta, F. Karsch, P. Petreczky and I. Wetzorke, Phys. Rev. D69 (2004) 094507.

[24] T. Umeda, K. Nomura and H. Matsufuru, Eur. Phys. J. C 39S1 (2005) 9.

[25] A. Jakovac, P. Petreczky, K. Petrov and A. Velytsky, hep-lat/0603005.

[26] H. Iida, T. Doi, N. Ishii, H. Suganuma and K. Tsumura, hep-lat/0602008.

[27] T. Hatsuda, Int. J. Mod. Phys. A 21 (2006) 688 [hep-ph/0509306].

[28] G. Aarts, C. R. Allton, R. Morrin, A. P. O. Cais, M. B. Oktay, M. J. Peardon and J. I. Skullerud, hep-lat/0610065.

[29] S. Datta, F. Karsch, S. Wissel, P. Petreczky and I. Wetzorke, hep-lat/0409147. 
[30] G. Aarts, C. Allton, J. Foley, S. Hands and S. Kim, hep-lat/0610061.

[31] M. Asakawa, T. Hatsuda and Y. Nakahara, Nucl. Phys. Proc. Suppl. 119 (2003) 481; Prog. Theor. Phys. Suppl. 149 (2003) 42.

[32] S. Datta, A. Jakovac, F. Karsch and P. Petreczky, hep-lat/0603002.

[33] S. Digal, O. Kaczmarek, F. Karsch and H. Satz, Eur. Phys. J. C 43 (2005) 71 [hep-ph/0505193].

[34] A. Mocsy, hep-ph/0606124.

[35] C. Y. Wong, hep-ph/0606200.

[36] B. A. Gelman, E. V. Shuryak and I. Zahed, nucl-th/0601029; nucl-th/0605046. 